\section{Leukemia takes it up a NOTCH}

\section{By Lev Osherovich, Senior Writer}

The Notch pathway has been linked to aggressive growth in a range of tumor types but had not previously been considered a prime target for myeloid leukemia therapies. Separate American teams now have identified two targets in the pathway that promote highly aggressive hematopoietic stem cell growth and possibly progression of late-stage chronic myeloid leukemia into acute myeloid leukemia. The work opens new therapeutic areas to the companies targeting the pathway for solid tumors.

Cancer stem cells are self-renewing, undifferentiated cells that are thought to fuel tumor growth. Ordinarily, stem cells divide to produce a copy of the original cell plus a daughter cell with some degree of differentiation, but cancer stem cells can divide without undergoing differentiation.

"A normal stem cell needs to decide whether to remain a stem cell or to differentiate," said Michael Kharas, instructor of hematological
"We found that MSI2 was massively upregulated as disease went from chronic to acute phases. This is consistent with the idea that MSI2 suppresses NUMB homolog."

- Tannishtha Reya,

Duke University School of Medicine

\section{Having a blast}

Reya's team created cancerous human hematopoietic stem cells and transplanted them into mice. Compared with early-stage CML cells, NUMB homolog was underexpressed in CML cells undergoing blast crisis, an aggressive late stage of the disease that resembles AML.

"We looked at leukemia progression from chronic to acute phase. The chronic phase is manageable but the acute phase grows very fast," she said.

NUMB homolog is a developmental factor in flies, but in leukemia it acts like a tumor suppressor. Indeed, mice transplanted with blast crisis cells that overexpressed NUMB homolog had better survival than mice with cells that lacked the protein.

Because numb is regulated by musashi in flies, Reya's team examined levels of Musashi family members in human blast crisis CML cells and found that MSI2 was overexpressed compared with its expression in low-growing CML controls.

The team went on to show that knocking down Msi2 slowed tumor growth and increased survival in a mouse model of blast crisis CML.

"We found that MSI2 was massively upregulated as disease went from chronic to acute phases," said Reya. "This is consistent with the idea that MSI2 suppresses NUMB homolog."

\section{Comfortably numb}

Separately, a team led by Kharas and George Daley came across MSI2 while studying gene expression in normal hematopoietic stem cells (HSCs) in mice.

Daley is professor of hematology and direconcology at Brigham and Women's Hospital. "This decision point could be mutated in cancers."

Kharas is the lead author of a study published in Nature Medicine that identified the pathway's role in AML. ${ }^{1}$ The other report showed the pathway's involvement in CML and was published in Nature by a team led by Tannishtha Reya, associate professor of pharmacology and cancer biology at Duke University School of Medicine. ${ }^{2}$

Kharas noted that in cancer, inappropriate activation of developmental pathways such as Notch signaling can cause stem cells to divide symmetrically to produce two more stem cells. Excess accumulation of these stem cells is a hallmark of many aggressive tumors. Moreover, the stem cells are hard to eradicate with conventional chemotherapy because they lack many of the targets and processes that drive the growth of the more differentiated cancer cells.

One new target, NUMB homolog, inhibits notch homolog 1 translocation-associated (NOTCH1) processing and activation. The other target, an RNA-binding protein called Musashi homolog 2 (MSI2), blocks the translation of NUMB homolog. The former could be therapeutically activated whereas the latter one could be inhibited.

Both targets offer an opportunity to intervene upstream of other Notch pathway targets, but hitting these intracellular proteins may be challenging. tor of the stem cell transplantation program at Children's Hospital Boston and professor of biological chemistry, molecular pharmacology and pediatrics at Harvard Medical School.

The Boston team found that HSCs with high MSI2 expression were less differentiated and more stem cell-like than bone marrow cells with low MSI2 expression.

Previous studies have linked a homolog of MSI2 called MSI1 to solid tumor progression. ${ }^{3}$ Thus, Kharas and Daley tested whether MSI2 expression affected leukemia progression in mice. Their group found that MSI2 overexpression alone did not cause leukemia. However, combining excess MSI2 with a BCR-ABL oncogene, a common cause of CML, dramatically increased tumor proliferation compared with using only BCR-ABL.

The team also found that AML patients with high levels of MSI2 had faster-growing tumors and worse survival rates than patients with low MSI2 levels.

Mechanistically, the findings show that excess MSI2 leads to degradation of NUMB homolog mRNA, which in turn removes an impediment to the proteolytic processing of NOTCH1 by $\gamma$-secretase (see Figure 1, "Musashi homolog 2 and NUMB homolog in cancer").

The studies also suggest that increased MSI2 can drive CML into an aggressive AML-like state and that many AML patients have high MSI2 
levels. Thus, it is possible that AML patients with increased MSI2 may have started off with CML that shifted into overdrive due to MSI2 overexpression.

“Though we can't prove that all AML cases result from CML conversion via MSI2, a large majority of the patients have high MSI2 expression" in the most aggressive forms of both disorders, said Kharas.

Reya and Kharas also noted that other cancer pathways including tumor protein p53 (TP53; p53) and Hedgehog signaling may be regulated by MSI2. Both teams are working to identify other downstream targets of MSI2 besides NUMB homolog.

In addition, both Kharas and Reya think screening for increased MSI2 levels could be useful for catching the emergence of aggressive leukemias.

\section{Not your average target}

Although many of the players in the Notch pathway are familiar from previous developmental and cancer studies, the leukemia connection opens up new opportunities for companies developing antagonists of NOTCH1 signaling in hematological malignancies.

At least nine companies have compounds against various targets involved in transcriptional activation by the Notch pathway in preclinical and clinical development for solid tumors.

"MSI2 has not previously been known for its relevance to hematopoiesis", said Kristin Hope, associate professor of biochemistry and biomedical sciences at McMaster Stem Cell and Cancer Research Institute.

Hope and colleagues reported in Cell Stem Cell last month that

DLL4 inhibitors: OMP-21M18 in Phase I for solid tumors from OncoMed Pharmaceuticals Inc./GlaxoSmithKline plc (LSE:GSK; NYSE:GSK); REGN421 (SAR153192) in Phase I for solid tumors from Regeneron Pharmaceuticals Inc.

(NASDAQ:REGN)/sanofi-aventis Group (Euronext:SAN; NYSE:SNY)

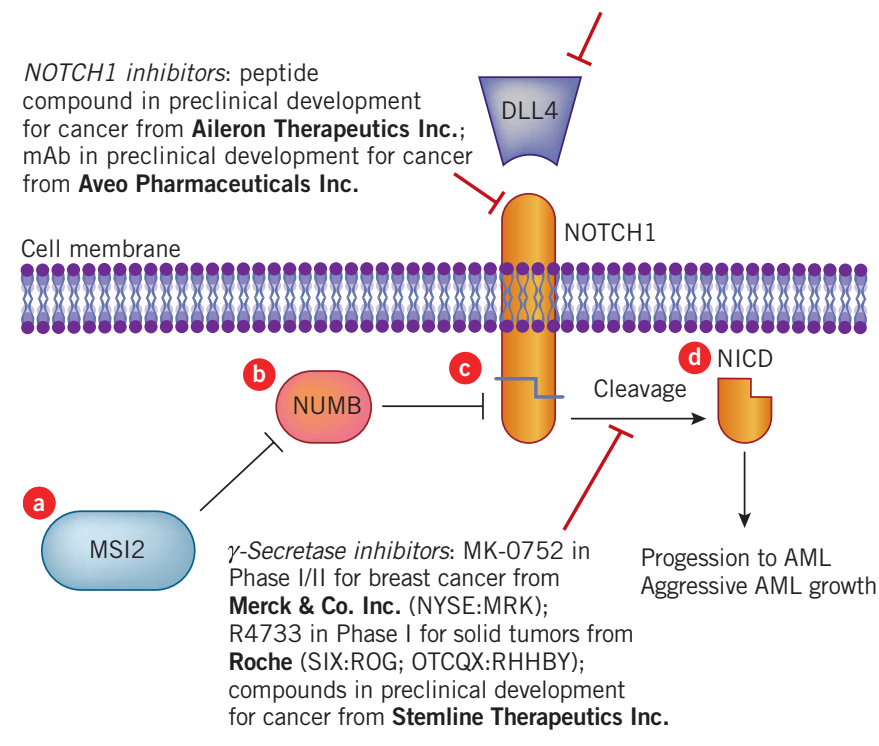

MSI2 is one of several factors involved in stem cell division. ${ }^{4}$

"If MSI2 is upregulated in CML, this implies that NUMB homolog is downregulated and Notch signaling is upregulated," said Timothy Hoey, SVP of cancer biology at OncoMed Pharmaceuticals Inc. "This highlights CML as a potential opportunity for our NOTCH pathway antagonists."

He added that turning down NOTCH1 signaling "increases sensitivity to chemotherapeutic agents and reduces tumor recurrence and metastasis."

OncoMed's OMP-21M18, a mAb that targets the NOTCH1 activator delta-like ligand 4 (DLL4), has completed Phase I testing in solid tumors. The company plans to submit an IND for a mAb targeting NOTCH1 itself this year.

"The issue of self-renewal is central to cancer and is closely related to the asymmetric division" that is governed by MSI2, said Tom Cirrito, director of operations at Stemline Therapeutics Inc. He thinks blocking the action of MSI2 or its downstream components in the Notch pathway therefore could correct the imbalanced symmetric division that leads to cancer stem cell production.

"Symmetric cell division maintains the pool of cancer stem cells," he said. "We would want compounds that promote differentiation and get rid of cancer stem cells. These papers point to symmetric cell division as a process that can be targeted" to treat cancer.

Stemline has two $\gamma$-secretase inhibitors in preclinical development for solid tumors: SL301 and SL302. Stemline's lead program is SL401, a peptide that targets IL-3 receptor (CD123). The compound is in Phase I testing to treat AML.

Figure 1. Musashi homolog 2 and NUMB homolog in cancer. Ito et al. and Kharas et al. have identified a pathway that activates Notch homolog 1 translocation-associated (NOTCH1) and promotes progression of chronic myeloid leukemia $(\mathrm{CML})$ to acute myeloid leukemia (AML).

The RNA-binding protein Musashi homolog 2 (MSI2) [a] is overexpressed in blast crisis-stage CML cells and in AML cells. MSI2 blocks the translation of mRNA encoding Numb homolog (NUMB) [b]. Because NUMB normally inhibits the proteolytic cleavage and activation of NOTCH1 [c], low NUMB levels lead to higher levels of the NOTCH1 intracellular domain (NICD) [d], which activates genes involved in aggressive leukemia growth.

A number of cancer compounds are in development that target proteins in the pathway, including NOTCH1 and its ligand, delta-like ligand 4 (DLL4), and $\gamma$-secretase, an enzyme involved in the processing of NOTCH1 into NICD. 


\section{ANALYSIS}

\section{TARGETS \& MECHANISMS}

Although blocking MSI2's activity or increasing levels of NUMB homolog should produce therapeutic effects, targeting these intracellular proteins will not be easy.

"MSI2 is an RNA-binding protein, not a kinase, so it's very hard to get at," said Reya. One tactic for antagonizing MSI2 might be to study the "post-translation modifications that regulate its function or its downstream targets," she added.

Besides trying to knock down MSI2 with small interfering RNA, Cirrito suggested making an RNA mimetic that binds and blocks MSI2's RNA-binding site. The effect of such an oligonucleotidebased agent would be to prevent the degradation of NUMB homolog mRNA and other MSI2 targets, he noted.

Both Kharas and Reya have filed for patents on diagnostic and therapeutic applications of their discoveries. The IP is available for licensing.

Osherovich, L. SciBX 3(32); doi:10.1038/scibx.2010.974

Published online Aug. 19, 2010

\section{REFERENCES}

1. Kharas, M.G. et al. Nat. Med.; published online July 8, 2010; doi:10.1038/nm.2187

Contact: George Daley, Brigham and Women's Hospital, Boston, Mass. e-mail: george.daley@childrens.harvard.edu

Contact: Michael Kharas, same affiliation as above e-mail: mkharas@partners.org

2. Ito, T. et al. Nature; published online July 19, 2010; doi:10.1038/nature09171

Contact: Tannishtha Reya, Duke University School of Medicine, Durham, N.C. e-mail: t.reya@duke.edu

3. Sureban, S.M. et al. Gastroenterology 134, 1448-1458 (2008)

4. Hope, K.J. et al. Cell Stem Cell 7, 101-113 (2010)

COMPANIES AND INSTITUTIONS MENTIONED

Brigham and Women's Hospital, Boston, Mass.

Children's Hospital Boston, Boston, Mass.

Duke University School of Medicine, Durham, N.C.

Harvard Medical School, Boston, Mass.

McMaster Stem Cell and Cancer Research Institute, Hamilton, Ontario, Canada

OncoMed Pharmaceuticals Inc., Redwood City, Calif. Stemline Therapeutics Inc., New York, N.Y. 